\title{
PAPER
}

\section{Distinct neuropsychological characteristics in Creutzfeldt-Jakob disease}

\section{J S Snowden, D M A Mann, D Neary}

See Editorial Commentary pages 613-14

J Neurol Neurosurg Psychiatry 2002;73:686-694

Objectives: To characterise the nature of cognitive change in Creutzfeldt-Jakob disease (CJD).

Methods: Case histories are reported of four patients with sporadic (sCJD) and two with familial CJD (fCJD), with postmortem pathological findings in four cases. The data derived from cognitive examina-

See end of article for authors' affiliations

.....................

Correspondence to: Dr J S Snowden, Cerebral Function Unit, Greater Manchester Neuroscience Centre, Hope Hospital, Salford M6 8HD, UK;

julie.snowden@man.ac.uk

Received 3 April 2002

In revised form 26 July

2002

Accepted 8 August 2002 tion are examined with respect to the presence or absence of a variety of characteristics to elicit performance profiles across cognitive domains.

Results: Three patients with sCJD exhibited clear focal cortical deficits. One patient had visual impairment leading to cortical blindness, associated with posterior hemisphere abnormalities on single photon emission computed tomography (SPECT) imaging; two others had impairments in language, mirrored by left hemisphere SPECT abnormalities. The remaining three patients showed no specific cortical symptomatology. Despite these differences all six patients shared common qualitative characteristics: episodic unresponsiveness, interference effects, and profound verbal and motor perseveration. These common features are interpreted in terms of impaired activation and regulation of neocortex from subcortical structures. Findings from postmortem pathological examination and from the published literature provide converging evidence to implicate a critical role of the thalamus.

Conclusion: These preliminary findings suggest that $\mathrm{SCJD}$ and FCJD may be associated with distinct neuropsychological characteristics.
C ognitive impairment is a central feature of CreutzfeldtJakob disease (CJD), in both its sporadic and familial forms, ${ }^{1-5}$ and constitutes a core component of clinical diagnostic criteria..$^{6-8}$ Nevertheless clinical reports suggest that the nature of the earliest cognitive change is highly variable. Some patients present with focal cortical deficits such as aphasia ${ }^{9-11}$ or visual disturbance, ${ }^{1-5}{ }^{12}$ whereas early symptoms in other patients are less specific and include forgetfulness, poor concentration, lethargy, malaise, and apprehension. ${ }^{23}$ It has been assumed that a generalised dementia invariably ensues, traditionally thought to be without characteristic features and indistinguishable from more common forms of dementia such as Alzheimer's disease. ${ }^{2}$ The prevailing notion therefore is that there is no distinct profile of cognitive change characteristic of CJD.

Despite such traditional assumptions systematic study of cognitive change in sporadic CJD (SCJD) and familial CJD (fCJD) has been severely limited. The paucity of studies is unsurprising. CJD is rare, so that few centres accumulate patient cohorts of substantial size. Moreover, the rapidity of the disease course, particularly in $\mathrm{SCJD}$, and the severity of disease renders many patients formally untestable by standard psychological instruments by the time of their hospital admission. Untestability does not, however, necessarily signify an undifferentiated pattern of cognitive impairment. We have had the opportunity to examine a consecutive series of six patients with CJD, four with SCJD and two with fCJD, one presumed. Their illness was sufficiently severe to render impractical the administration of a lengthy battery of neuropsychological tests. They were administered a locally developed neuropsychological screening instrument, lasting about one hour, which has been found useful in the characterisation of forms of dementia. ${ }^{13}{ }^{14}$ The instrument examines a range of language, perceptual, spatial, praxic, memory, and executive skills, and is designed to provide a profile of strengths and weaknesses across cognitive domains. Emphasis is given to qualitative characteristics of test performance and the nature of errors. ${ }^{14}$ Although the six patients differed substantially in terms of the nature of presenting symptoms and the dominant domain of cognitive impairment we identified characteristics that were common to all patients, and were distinct from those seen in other forms of dementia. We present a series of case reports, examine the nature of the neuropsychological disorder, and consider its underlying basis.

\section{CASE REPORTS}

The six patients were consecutive referrals to a neurological department; in five, the clinical diagnosis was supported by pathological findings, and in three, by genetic analysis. Tables 1 and 2 show demographic and clinical characteristics. Cases 2 and 5 are likely to be sporadic in view of the absence of family history, but as no genetic analysis was performed this is presumptive rather than definitive.

\section{Patient 1}

Historical background

At the age of 51 this joiner complained of disturbed vision. Rooms in his home looked different and unfamiliar, and furniture appeared to move and distort. The luminance of the room would suddenly alter, as if someone had switched off the light. His wife noticed him to be jumpy, fearful, and withdrawn. She also noted that he tended to neglect his left arm, which he would hold in mid-air for prolonged periods. Following initial referral to an ophthalmologist he was admitted for neurological investigation three months after the onset of symptoms.

Abbreviations: CJD, Creutzfeldt-Jakob disease; $C T$, computed tomography; EEG, electroencephalogram; fCJD, familial Creutzfeldt-Jakob disease; sCJD, sporadic Creutzfeldt-Jakob disease; SPECT, single photon emission computed tomography; vCJD, new variant Creutzfeldt-Jakob disease 
Table 1 Patient demographics

\begin{tabular}{lllllll}
\hline & & & \multicolumn{3}{l}{ Illness duration at } \\
Patient & CJD subtype & Sex & $\begin{array}{l}\text { Age at } \\
\text { onset }\end{array}$ & $\begin{array}{l}\text { first assessment } \\
\text { (months) }\end{array}$ & $\begin{array}{l}\text { Total illness } \\
\text { duration (months) }\end{array}$ & $\begin{array}{l}\text { Pathological } \\
\text { confirmation }\end{array}$ \\
\hline 1 & Sporadic & M & 51 & 3 & 4 & Yes \\
2 & Sporadic & M & 55 & 11 & 14 & Yes \\
3 & Familial & F & 56 & 6 & 8 & Yes \\
4 & Familial & M & 59 & 2 & 3 & No \\
5 & Sporadic & F & 34 & 6 & 9 & Yes (biopsy) \\
6 & Sporadic & F & 59 & 6 & 9 & Yes \\
\hline
\end{tabular}

Table 2 Presenting features and investigations

\begin{tabular}{lllllll}
\hline Patient & Presenting symptom & $\begin{array}{l}\text { EEG diffuse } \\
\text { slowing }\end{array}$ & $\begin{array}{l}\text { EEG } \\
\text { triphasic }\end{array}$ & CT & $\begin{array}{l}\text { SPECT } \\
\text { abnormality }\end{array}$ & $\begin{array}{l}\text { SPECT } \\
\text { emphasis }\end{array}$ \\
\hline 1 & Visual disorder & + & + & Normal & Multiple & Posterior \\
2 & Anomia & + & + & Normal & Multiple & Left anterior \\
3 & Agraphia & + & + & Normal & Multiple & Left \\
4 & Personality change & + & - & Atrophy & Patchy & Left anterior \\
5 & Poor concentration & + & + & Normal & n.a. & n.a. \\
6 & Unsteadiness, poor memory & + & + & Atrophy & n.a. & n.a. \\
\hline
\end{tabular}

+, abnormality present; -, abnormality absent.

Neurological findings and investigations

He had a full range of voluntary eye movements and intact pupillary responses, but he was effectively blind. Power was preserved but there was rigidity of all four limbs with catatonic posturing of the arms. Tendon jerks were brisk, the left plantar response was extensor. There were no grasp reflexes. He tended to ignore tactile stimuli applied to the left limbs. His gait was ataxic. There were multiple myoclonic jerks of all four limbs. Successive electroencephalograms (EEGs) showed markedly increasing abnormalities with diffuse slow wave activity and transient bursts of slow and sharp waves. Computed tomography (CT) brain scan was normal but single photon emission computed tomography (SPECT) scan showed several areas of reduced uptake, particularly in the posterior regions.

\section{Cognitive evaluation}

He looked anxious and perplexed, and stared into space, or else his gaze wandered. His ability to cooperate in testing was variable. There were marked episodic fluctuations in attention, with brief intervals of seconds or sometimes minutes duration during which he was uncommunicative, unresponsive, and inaccessible to testing, interspersed between periods of responsiveness and appropriate engagement in tasks. His responsiveness was facilitated by his wife's presence and by discussion of personally and emotionally relevant topics. Response latencies during responsive periods varied from rapid to prolonged.

During periods of optimal responsiveness he was fully orientated in time and place. There were no primary disturbances of language. He produced grammatically correct sentences without paraphasic error. Comprehension of both nominal terms and functional words was good and he could respond correctly to syntactically complex questions. He had no difficulty repeating sentences. Naming of common objects from verbal description was quick and accurate. He showed good use of symbolic gesture and action pantomime. Memory of both autobiographical and news events was surprisingly well preserved, particularly for sports events for which he had particular interest. He recognised famous names, contemporary, past, and historic and provided appropriate identifying information. Visual tasks were, however, impossible. He showed no visual fixation on objects and he reported only seeing light which would suddenly become dimmed. During responsive periods he was insightful, and would give a verbal commentary on his visual experiences (for example, "The room's gone dark", and later "It's gone light and back to normal again"). He exhibited impressive powers of self reflection and would attempt to analyse and rationalise his presenting visual symptoms (for example, "Things seemed to move when I looked at them too long. So I kept moving my eyes to stop it happening").

Nevertheless, his test performance varied over the course of a single testing session and despite the absence of primary aphasia, apraxia, or amnesia, there were qualitative abnormalities across all tasks. Although linguistically correct his responses to questions were often irrelevant in content. Perseveration of his own earlier responses and intrusions from the interviewer's earlier remarks were frequent. Sometimes, he did not respond to the current question, but instead responded to an earlier question, which had been put to him during a period of inaccessibility and to which he had failed at the time to respond. Motor performance was also hampered by marked perseveration.

\section{Progression}

His condition deteriorated rapidly, with the unresponsive periods becoming more frequent and prolonged and he died two weeks after initial evaluation. In the terminal phase he was totally inaccessible and there was severe myoclonus. Postmortem examination was undertaken.

\section{Pathology}

The brain appeared normal, both externally and on coronal section, except for a mild and patchy atrophy within the posterior parietal and occipital lobes together with a mild bilateral enlargement of the posterior lateral ventricle. A slight état lacunaire within the basal ganglia was present. The grey matter of the cerebral cortex showed for the most part only slight spongiform change, together with a fine and patchy radial astrocytosis. However, the primary visual and visual association cortex also showed, along with the mild spongiosis, a severe reactive astocytosis in subpial and pyramidal cell layers; within the underlying white matter a mild astrocytosis was present. The corpus striatum and thalamus showed only a mild spongiosis and gliosis. The cerebellar cortex showed mild spongiosis, especially at the tips of the folia, though there was a moderate to severe radial gliosis. The granule cell layer and 
the dentate nucleus appeared normal, though a patchy loss of Purkinje cells was seen. The deep white matter of the cerebellum was mildly gliotic. No deposits of prion protein as amyloid plaques were present in any part of the brain.

Prion protein and gene analysis

Immunoblotting and Western blotting revealed the presence of the abnormal isoform of prion protein. Sequencing the open reading frame of the prion gene revealed no mutations. Codon 129 showed methionine homozygosity.

\section{Patient 2}

Historical background

At the age of 55 this engineer developed increasing difficulties in naming and he adopted a strategy of drawing to facilitate communication. Six months later his writing deteriorated, with spelling errors and letter omissions and he could no longer sign his name. However, his comprehension, both aural and written, was regarded as intact. Three months later he was noted to have difficulty carrying out sequential activities. When making coffee he poured water into the coffee jar instead of the cup; when lighting his pipe he had difficulty judging where to apply the flame, and he had been noted to put one unlit pipe in his mouth while lighting another in his hand. His ability to identify faces and objects appeared intact, but spatial abilities such as dressing and telling the time were impaired. Memory for day to day and current news events was variable. His personality was regarded as unchanged and he showed introspection and appropriate concern regarding his illness.

\section{Neurological findings and investigations}

Neurological examination, carried out 11 months after onset of illness, revealed intact visual fields but some difficulty in visual localisation. Eye movements were slow and convergence was limited. There was muscular rigidity, brisk tendon reflexes, equivocal plantar responses, and bilateral grasp reflexes. Movements were accurate but perseverative and synkinetic. He was noted intermittently to maintain fixed postures of the limbs and trunk for several minutes, during which time he was mute.

The electoencephalogram displayed generalised delta wave activity with triphasic wave forms over the right cerebral hemisphere. Computed tomography was normal. SPECT imaging revealed multiple areas of reduced uptake in the cerebral cortex, especially in the left frontal lobe.

\section{Cognitive evaluation}

Evaluation of his mental state revealed variability in his level of responsiveness, with periods of total unresponsiveness of seconds or minutes duration. Sometimes he appeared vacant and perplexed and he either failed to respond at all or else his responses were irrelevant in content. At other times he responded appropriately and showed surprising flashes of insight into his illness and its traumatic effect on his family. The day prior to the assessment he had wandered from the hospital and had been found by the police, but was unresponsive when questioned by them. During the course of the following day's assessment he spontaneously commented how frightened he had been when alone in the city, his worries of being mugged, and his sense of relief when the police arrived. He remarked also on the oddity of being unable to communicate with the police and pondered the basis for this difficulty. His rate of both verbal and motor responses during the course of the assessment interview was excessively slowed.

His language was normal in grammatical structure, paraphasias were absent, and he made good use of affective prosody. However, his utterances were characterised by perseveration and disjointed, stereotyped phrases, chiefly referring to autobiographical events. Intrusions both from earlier conversations and from environmental stimuli occurred: names of nearby objects were interpolated irrelevantly into his speech output. Assessment of comprehension was complicated by the variability and perseverative nature of both verbal and motor responses, but there was no convincing evidence of a primary impairment at a lexical-semantic level. However, he had difficulty understanding complex syntax. His repetition span was reduced to four digits. Confrontation naming was markedly perseverative and verbal fluency yielded no response. Naming performance for individual items was variable across repeated naming trials. Reading and spelling were impaired by perseverations and intrusions from earlier tasks. Writing could not be attempted. Perceptual identification skills were difficult to evaluate because of a profound difficulty in spatial localisation of stimuli and distraction from irrelevant environmental stimuli. In carrying out gestural and nonrepresentational actions and in motor sequences and drawing there was synkinesis and marked perseveration. Memory could not be formally assessed. However, on some occasions he appeared disorientated and could access little relevant autobiographical information, whereas on others he gave a coherent account of events. He was noted to be most responsive and communicative when the topic of conversation related to his personal and family life and was of emotional significance to him.

He became increasingly prone to periods of inaccessibility over the ensuing three months until his death, with increasingly prolonged periods of limb posturing and frequent startle reaction to noise. Postmortem brain examination was undertaken.

\section{Neuropathological changes}

The brain appeared normal, both externally and on coronal section. The grey matter of the cerebral cortex showed a widespread and severe spongiosis extending throughout all cortical layers. There was extensive loss of nerve cells and vacuolation of remaining cells, though reactive astrocytosis was slight. The corpus striatum showed similar changes, though within the thalamus spongiosis was less extensive but a moderate gliosis was present. The cerebellar cortex showed a mild spongiform change with moderate gliosis of both grey and deep white matter. Granule and Purkinje cells were normal. No deposits of prion protein in the form of amyloid plaques were present in any part of the brain.

\section{Patient 3}

\section{Historical background}

At the age of 56 this remedial teacher noticed difficulty in writing, both in spelling and in executing individual letters. Four months after onset of symptoms she ceased to write, and exhibited difficulties in oral expression. Speech was hesitant, stuttering, and contained phonemic errors, of which she was aware. She complained of problems in word finding. During the next month she became unable to dress and orientate clothing. She was insightful and became anxious and distressed. She was admitted for investigation six months after onset of symptoms.

\section{Neurological findings and investigations}

Neurological examination revealed intact vision. Facial expression was slightly reduced. There was increased tone and minimal weakness of the right arm and leg. She exhibited posturing of the right arm. The tendon reflexes were brisker on the right side and both plantar responses were extensor. Elementary sensation in the limbs was preserved, but she had marked clumsiness of both hands and difficulty replicating hand postures. She walked slowly with reduced arm swing. There were multiple myoclonic jerks of the limbs.

Electroencephalography revealed diffuse slow wave activity on which was superimposed recurring runs of higher voltage delta activity with some triphasic waves. The CT was normal. 
The SPECT scan revealed abnormal uptake in both cerebral hemispheres and especially the left cerebral cortex.

\section{Cognitive evaluation}

On mental examination she appeared apprehensive and preoccupied by her communication difficulties. Her social skills were well preserved. Her speech was hesitant and effortful and contaminated by profound perseveration of phrases and prior item intrusions. Phonemic paraphasias also occurred, which she would attempt to rectify by successive attempts. Overlearnt verbal series, once initiated were produced with relative ease. Evaluation of comprehension indicated relatively good understanding at the single word level, but poor comprehension of complex syntactic sentences. Repetition, naming, reading, and writing were grossly perseverative. Perceptual and spatial skills were less affected than her language skills. She was able to fixate and recognise objects. Motor actions, both symbolic and nonrepresentational, were severely compromised by perseveration. Memory assessment was constrained by her expressive difficulties. Nevertheless, she was orientated and could provide information about autobiographical events. She was able to describe, within the limits of her expressive powers, the course of her illness and the nature of her symptoms.

\section{Progression}

With progression of disease, variability in her performance was increasingly evident, with periods during which she was unable to respond interspersed with periods during which she was relatively communicative. She became increasingly distractible, both by visual and auditory stimuli. She deteriorated rapidly, the periods of unresponsiveness becoming more frequent and prolonged. Verbal expression became limited to a few perseverative, stereotyped phrases and finally ceased. Dyspraxia affected all manual tasks and whole body movements. Myoclonus was marked. She died six weeks after her initial hospital investigation. Postmortem brain tissue examination was carried out.

\section{Neuropathological changes}

The brain appeared normal externally and on coronal section only a mild ventricular enlargement was seen. A severe loss of nerve cells and a profound spongiosis was present throughout the cortical laminae within the frontal convex cortex and occipital cortex; the orbitofrontal cortex, insular, temporal, and parietal cortex showed less change. An intense reactive astrocytosis was present in all cortical grey and white matter areas, irrespective of whether spongiosis was present or not. The putamen showed a severe loss of neurones, spongiosis, and gliosis, though the caudate nucleus and thalamus were less severely affected. The cerebellar cortex showed minimal spongiosis, though there was a moderate radial gliosis in the molecular layer with some gliosis in Purkinje and granule cell layers. The deep white matter was intensely gliotic. There was patchy loss of both Purkinje and granule cells.

Many amyloid plaques composed of prion protein were present in the frontal cortex, cerebellum, and putamen, and to a lesser extent in other cortical areas, caudate nucleus, and thalamus. In the cerebellum they were mostly present in the molecular layer, though others were seen in granule and Purkinje cell layers. The plaques were of a single or multicentric amorphous type, as commonly seen in GerstmannStraussler-Sheinker syndrome, with occasional "clouds" of deposit throughout the depth of the molecular layer. Similar globular, multicentric, or conglomerate deposits were present in the frontal cortex. The plaques were heavily invested with astocytic processes, though little microglial activity was present.

Immunoblotting and Western blotting revealed the presence of the abnormal isoform of prion protein. Sequencing the open reading frame of the prion gene revealed proline to leucine mutation at codon 102. Codon 129 was methionine/valine heterozygous.

\section{Patient 4}

Historical background

This 59 year old welder underwent rapid personality change with increased aggression, together with slurring of speech and difficulty walking. There was a very rapid physical decline, leading to his hospital admission two months later.

\section{Neurological findings and investigations}

On examination, his speech was dysarthric and he had weakness and increased tone in the lower limbs, which tended to be held in flexion. Tendon reflexes were brisk and both plantar responses were extensor. Elementary sensation was intact. He had an ataxic gait and there were multiple myoclonic jerks of the limbs. Computed tomography revealed mild cerebral atrophy. The EEG showed gross delta wave activity but without phasic complexes. SPECT showed patchy reductions in tracer uptake, affecting the left hemisphere more than the right.

\section{Cognitive evaluation}

He looked frightened and bewildered. He displayed striking fluctuations in his level of responsiveness. For the majority of the time he was totally unresponsive. During these prolonged periods he was speechless, but restless, making repetitive movements with the hands or adopting fixed postures of the limbs. However, there were brief intervals of a few minutes duration during which he was responsive. In these periods he spoke comprehensibly without paraphasias. He read aloud a short story accurately. He recognised and named common objects. He completed simple written additions. He copied gestures and non-representational hand postures accurately and he participated in games of noughts and crosses, showing both spatial awareness and preserved knowledge of the rules of the game. Nevertheless, both verbal and motor responses were marred by marked perseveration and execution of tasks was slowed.

It was noted that in the presence of clinicians he was unresponsive for most of the time, failing to attend to stimuli shown to him and failing to respond to questions either verbally or by gesture. Nevertheless, in the company of his family he was noted to be responsive and communicative for prolonged periods.

\section{Progression}

He continued to decline rapidly, becomingly increasingly and then totally unresponsive and died three months after onset of symptoms. Postmortem examination was not performed. However, a similar rapidly progressive dementia occurred in his brother and a cousin, and in the latter there was postmortem pathological confirmation of the diagnosis of CJD.

\section{Patient 5}

\section{Historical background}

At the age of 34 this housewife complained of impaired memory and loss of confidence. Three months after onset of symptoms she was slow and vague in replies and disorientated, and became increasingly anxious and distressed. She was referred to a psychiatrist who noted the variable and patchy nature of her amnesia and disorientation. The variability, together with her physical wellbeing, a normal computed tomogram, and the presence of major domestic problems led to a diagnosis of non-organic illness. However, her symptoms progressed, leading to a neurological referral three months later.

Neurological findings and investigations

Neurological examination revealed minor disturbances only. There was dyskinesia of the jaw, a mild increase in tone of the 
upper limbs, and brief brisk myoclonic jerks on startle. Computed tomography was normal. The electrocephalogram revealed diffuse excess of slow wave activity, episodic in nature.

\section{Cognitive evaluation}

Her mental performance was characterised by variable responsiveness. Usually she appeared bewildered and remained silent or produced stereotyped phrases, perseverated earlier responses, or echoed the examiner's question. However, periodically she produced pertinent replies, which were grammatically correct and without paraphasias; she named objects and famous faces, showed comprehension of simple verbal instructions, and responded appropriately to action commands. Her memory could not be assessed because of her unresponsiveness. During periods of optimal responsiveness she commented on the absurdity of her forgetfulness and expressed concern regarding her altered mental state.

\section{Progression}

Cortical biopsy was undertaken, confirming the diagnosis of CJD. She became increasingly unresponsive and died three months later.

\section{Patient 6}

Historical background

A 59 year old lady developed increasing unsteadiness leading to falls. She was noted by relatives to have problems with memory.

\section{Neurological findings and investigations}

On neurological examination six months after the onset of symptoms, muscular tone was increased but there was no weakness. Tendon jerks were brisk and plantar responses were flexor. Sensation was intact. There was a postural tremor and incoordination of the arms and the gait was ataxic. Startle myoclonus was observed. A CT brain scan revealed a minor degree of cerebral atrophy. The EEG was grossly abnormal with widespread delta wave activity.

\section{Cognitive evaluation}

Evaluation of her mental function revealed variability in her responsiveness. Frequently she was difficult to engage and unresponsive to questions and commands, whereas at other times she was alert and cooperative. When accessible to testing her speech was dysarthric, but there were no linguistic errors. Series speech was intact and she had a normal span of seven digits. Comprehension was good, both at the single word and sentence level and she showed good confrontation naming of objects and body parts. She could spell aloud, read, and write, although execution of script was compromised by incoordination. However, she had difficulty carrying out mentally effortful tasks involving generation, mental manipulation, and sequencing of information. Her verbal fluency performance was limited to one animal word and four " $\mathrm{F}$ " words in one minute. She could not produce the months of the year in reverse order; she had a reduced reverse digit span of 3 . She could not point to a sequence of four objects in the correct order. A similar pattern was seen with respect to non-language functions. There was no evidence of primary disorder of visual perception, spatial function, or praxis: she localised, identified, and manipulated objects normally, she replicated nonrepresentational hand postures, and she recognised and named faces of celebrities. However, she had difficulty grasping and accomplishing complex tasks such as block constructions. Drawings and manual sequences revealed perseveration. She was disorientated in time and place and was amnesic for personal autobiographical and current news events. During periods of optimal responsiveness she commented on the absurdity of her forgetfulness and expressed concern regarding her altered mental state.

\section{Progression}

Her condition deteriorated rapidly, periods of inaccessibility becoming more frequent and prolonged. She died of bronchopneumonia nine months after onset of symptoms. Postmortem examination was undertaken.

Neuropathological findings

The brain showed a mild and patchy atrophy, mostly within mid frontal and parietal lobes. On coronal section the angles of the lateral ventricles were rounded, though the latter were not much enlarged. A patchy and variable spongiosis and reactive astrocytosis was present throughout the cerebral cortex, but such changes were severe only in parts of the frontal and insular cortices. The caudate nucleus and putamen showed a particularly severe spongiosis and reactive astrocytosis, though less severe changes were present in the globus pallidus and medial thalamus. The cerebellar cortex showed a mild spongiosis within the molecular layer, though astrocytosis was severe. There was a patchy loss of granule cells and Purkinje cells with a mild astrocytosis within these latter areas. The deep white matter of the cerebellum was intensely gliotic. Prion plaques were widely present in the cerebellum, mostly in the Purkinje cell layer but also within molecular and granular cell layers. These were of a unicentric, "stellate" or "Kuru" type. Many similar plaques were present in the thalamus and corpus striatum, though none were seen in the cerebral cortex.

Immunoblotting and Western blotting revealed the presence of the abnormal isoform of prion protein. Sequencing in the open reading frame of the prion gene revealed no mutations, though codon 129 was methionine/valine heterozygous.

\section{NEUROPSYCHOLOGICAL PROFILE ANALYSIS}

The prospective cognitive evaluation of patients outlined in the case reports suggests that all patients were severely impaired at the time of their assessment. Nevertheless, far from showing an undifferentiated pattern of disorder, they appeared to show distinctive performance characteristics. Neuropsychological test scores alone, based on cut offs for pass or failure, would clearly fail to capture such qualitative characteristics and would be likely to submerge differences in the reason for test failure. Impaired naming scores, for example, might putatively reflect a primary linguistic disorder or might arise secondary to a general perseverative tendency not specific to the verbal domain. A retrospective analysis of data was undertaken, in an attempt to parcel out factors underlying patients' performance.

\section{Methods}

The presence or absence of a variety of performance characteristics was determined on the basis of written reports and analysis of patients' responses and error types. Retrospective analyses, particularly of qualitative data, have inevitably limitations. However, analyses were based on a common data set across all patients, reported in a standard format by a single neuropsychologist, suggesting the likelihood of internal consistency. The presence of each abnormality was determined as follows.

\section{Attention}

Variable responsiveness: documented report that the patient sometimes fails to respond, or there is an uncharacteristic time lag between question and initiation of response (that is, out of keeping with the usual rate of response), during which the patient is immobile with fixed gaze.

Distracted by visual environment: documented report that the patient's gaze wanders on to irrelevant stimuli or that the patient incorporates irrelevantly into his/her utterances names of objects or words from the visual environment. 


\section{Language}

Irrelevant content/intrusions: documented record of responses that are unrelated to question. These may reflect an intrusion from an earlier topic of conversation or an extraneous environmental stimulus.

Verbal perseverations: repetition of the preceding response. Based on spontaneous speech utterances and object naming responses.

Grammatical errors: based on analysis of recorded spontaneous speech utterances and test responses.

Semantic paraphasias: based on spontaneous speech utterances and object naming responses.

Phonemic paraphasias: based on spontaneous speech utterances, object naming responses, and repetition of sentences.

Impaired object naming: based on impaired naming of common objects, not accounted for by general unresponsiveness, intrusions, and perseverative responses. This includes lexical retrieval failures, and phonological and semantic naming errors.

Impaired lexical comprehension: based on patient's inability to point to a series of named objects and body parts.

Impaired syntactic comprehension: based on patient's inability to respond correctly to six syntactically complex sentences.

\section{Perceptuospatial abilities}

Impaired recognition of line drawings: based on 10 line drawings of common objects. Recognition failures are defined as those not attributable to general unresponsiveness, intrusions, or response perseverations. They include "don't know" responses and misperceptions.

Impaired recognition of faces: based on nine famous faces. Recognition failures are those not attributable to general unresponsiveness, intrusions, or response perseverations. They include "don't know" responses and misperception errors.

\section{Spatial skills}

Impaired localisation: based on documented reports of prolonged latencies in localising visual stimuli.

\section{Praxis}

Motor perseveration: based on documented report of perseveration of action in limb gesture, action pantomime, copying hand postures.

Impaired limb gesture and pantomime: impairment not accounted for by unresponsiveness or motor perseveration. This includes actions that are spatially degraded with respect to internal detail or overall position in space.

Impaired copy of non-representational hand positions: impairment not accounted for by unresponsiveness or motor perseveration. This includes actions that are spatially inaccurate with respect to internal detail or overall position in space.

\section{Memory}

Disoriented in time and place: incorrect orientation responses during periods of responsiveness, not accounted for by response perseverations. This includes both "don't know" and incorrect responses.

Impaired current autobiographical memory: inability to provide information about ongoing personal events, during periods of responsiveness.

\section{Executive skills}

Weigl's blocks: inability to sort blocks according to common feature (shape, colour, or motif) or identify sorting rule when blocks sorted by examiner.

Impaired verbal fluency: failure to generate more than five animals or " $\mathrm{F}$ " words in one minute.

\section{Behaviour}

Fearfulness, perplexity: based on documented report of patient's mental state.
Table 3 Cognitive and behavioural characteristics

\begin{tabular}{|c|c|c|c|c|c|c|}
\hline Abnormality & 1 & 2 & 3 & 4 & 5 & 6 \\
\hline \multicolumn{7}{|l|}{ Attention } \\
\hline Variable responsiveness & + & + & + & + & + & + \\
\hline Distracted by external environment & + & + & + & + & + & + \\
\hline \multicolumn{7}{|l|}{ Language } \\
\hline Irrelevant content/intrusions & + & + & + & + & + & + \\
\hline Verbal perseverations & + & + & + & + & + & + \\
\hline Grammatical errors in speech & - & - & - & - & - & - \\
\hline Semantic paraphasias & - & - & - & - & - & - \\
\hline Phonemic paraphasias & - & + & + & - & - & - \\
\hline Impaired lexical comprehension & - & - & - & - & - & - \\
\hline Impaired syntactic comprehension & - & + & + & $?$ & - & - \\
\hline Impaired object naming & - & + & + & - & - & - \\
\hline \multicolumn{7}{|l|}{ Perception } \\
\hline Impaired recognition line drawings & Blind & $?$ & - & - & - & - \\
\hline Impaired recognition famous faces & Blind & $?$ & $?$ & $?$ & - & - \\
\hline \multicolumn{7}{|l|}{ Spatial skills } \\
\hline Impaired localisation & Blind & + & - & - & - & - \\
\hline \multicolumn{7}{|l|}{ Praxis } \\
\hline Motor perseveration & + & + & + & + & + & + \\
\hline Impaired limb gesture and pantomime & - & + & - & - & - & - \\
\hline Impaired copy of hand postures & Blind & + & - & - & - & - \\
\hline \multicolumn{7}{|l|}{ Memory } \\
\hline Disoriented in time and place & - & + & - & $?$ & $?$ & + \\
\hline Impaired autobiographical events & - & - & - & $?$ & $?$ & + \\
\hline \multicolumn{7}{|l|}{ Executive } \\
\hline Weigl's block sorting & Blind & + & + & $?$ & $?$ & + \\
\hline Verbal fluency (animals/" $F$ " words) & + & + & + & + & + & + \\
\hline \multicolumn{7}{|l|}{ Behaviour } \\
\hline Fearfulness, perplexity & + & + & + & + & + & + \\
\hline Impaired self reflection & - & - & - & + & - & - \\
\hline Social disinhibition & - & - & - & - & - & - \\
\hline
\end{tabular}

Self reflection: based on documented report that patient comments on own illness/errors.

Social disinhibition: based on documented report of inappropriate social interaction.

\section{Results}

Table 3 shows the profile of performance in each patient. There were some features common to all patients. All showed variability in their responsiveness and all were distracted by their visual surroundings. In all, speech content was sometimes irrelevant, unrelated to the question asked. In all, perseverations dominated conversational speech and naming responses. No patient, however, made grammatical errors in conversation, none made semantic errors in naming, and none showed impairment in single word comprehension, at least for common objects.

Other language features varied across the group. Only patients 2 and 3 made phonological errors. In patient 2 these were elicited only on a repetition task, whereas in patient 3 there were sporadic phonemic paraphasias errors also in conversational speech and naming. Although perseverations dominated naming performance in all patients, only patients 2 and 3 provided evidence of naming impairment for common objects, beyond that attributable to general unresponsiveness or response perseveration. Both had difficulty comprehending complex syntactic sentences. Patients varied with respect to their performance on perceptual and spatial tasks. Patient 1 was effectively blind and thus was unable to carry out any task in a visual domain. Patient 2 showed impaired spatial localisation of objects, so that his perceptual recognition could not 
reliably be determined. Other patients were able to recognise line drawings and patients 5 and 6 identified and named famous faces. Motor perseveration dominated performance on manual tasks in all patients. In all, errors on tests of praxis predominantly represented perseverations of earlier actions. However, actions in patient 2 were also markedly spatially degraded and executed in an inappropriate part of space. Similarly, whereas copies of non-representational hand postures were spatially accurate in patients 3-6 (when not compromised by response perseveration) they were spatially degraded in patient 2 .

Memory performance was variable, both across patients and within patients. Only patient 6 was frankly amnesic, being disoriented in time and place and unable to provide any information about current autobiographical events. Patient 2 was disoriented on specific orientation questioning, yet gave a good account of ongoing events, commenting accurately on events of the previous days. Other patients showed good orientation and autobiographical memory, or else this could not be determined because of unresponsiveness to memory questions.

All patients showed highly impaired generational skills, and on a verbal fluency task no patient was able to produce more than five animal words in one minute or five " $F$ " words. All patients seemed bewildered, fearful, and perplexed. None was socially disinhibited.

\section{DISCUSSION}

The patients reported in this study differed in terms of their clinical presentation. Two sCJD and one fCJD patients presented with focal cortical deficits, patient 1 with alterations in primary vision, patient 2 with anomia, and patient 3 with difficulties in writing and spelling. The remaining three patients had a less specific pattern of symptomatology. The fCJD patient showed changes in behaviour, difficulty in walking, and slurring of speech. Two sCJD patients experienced poor concentration and variable memorising, lethargy, and malaise, together, in one patient, with unsteadiness. This heterogeneity mirrors other reports of SCJD. A wide spectrum of focal presentations have been reported that include aphasia, ${ }^{1-3}$ 9-11 $^{11}$ ataxia, ${ }^{3}{ }^{15} 16$ visual impairments, ${ }^{1-5}{ }^{12}$ cortical deafness, ${ }^{17-19}$ depression, ${ }^{20}$ and obsessive-compulsive disorder. ${ }^{21}$ In other patients the presentation is relatively nonspecific and may consist of lethargy and difficulty in concentration..$^{23}$

The six patients' clinical presentations were reflected by the pattern of findings on cognitive evaluation. Patient 1 was unable to attempt any task in a visual domain, yet he showed no evidence of primary impairment in language and he was not clinically amnesic. By contrast, patients 2 and 3 had notable language impairments, accompanied in patient 2 by problems in spatial localisation. The remaining patients showed no specific evidence of cortical symptomatology. They did not make linguistic errors, showed no problems in object or face perception, or in spatial localisation. The different performance profiles were reflected in findings on SPECT imaging. Abnormalities were most marked in the posterior hemispheres in patient 1 and in the left anterior hemisphere in patients 2 and 3 .

Despite heterogeneity with respect to the prominent domains of cognitive impairment there were qualitative performance characteristics common to all six patients. Firstly, and perhaps most strikingly, all patients showed fluctuations in the efficiency of their performance. Appropriate responding, with coherent, pertinent utterances, was interspersed by transient periods of unresponsiveness, during which the patient remained in a fixed position with a fixed gaze, but without evidence of drowsiness. Episodes of unresponsiveness varied in duration. They might be momentary, last several seconds, or persist over minutes. With progression of disease, periods during which the patient was inaccessible became increasingly prolonged and ultimately pervasive, leading to a state of akinetic mutism. Unresponsiveness did not imply an absence of information processing. In all patients there were instances in which a question posed during a period of unresponsiveness was, after a prolonged silence, answered correctly without the question having been repeated by the examiner. Patient 1 was noted on occasion to produce a pertinent response, not to the current question, but to an earlier question posed during a period of unresponsiveness. Patient 2 was able to provide an accurate commentary on incidents of the previous day during which he had been unresponsive, showing that he had registered ongoing events and acquired stable memories for them.

A second common feature was the presence of intrusion errors, arising both from auditory and visual stimuli. Some intrusions were within-test errors, constituting, for example, the repetition of a phrase spoken earlier by the examiner, an association with an earlier topic of conversation, or the provision of a response to an earlier question. Other intrusions, indicating visual distractibility, arose from irrelevant stimuli in the immediate visual environment. For example, a response to a question might include the name of an extraneous object on which the patient's attention happened to rest, or a word on a written notice. Intrusions influenced the quality of patients' spoken utterances. Although grammatically and linguistically correct, content was often irrelevant, unrelated to the question posed.

A third common feature was the presence of perseverative errors, occurring on both verbal and motor tasks. Perseverations were the dominant error type in naming tasks, even in patients 2 and 3 in whom language related symptoms constituted the presenting feature. They were also the dominant error type on manual tasks. Some perseverations had a distinctive and unusual quality, not encountered in other forms of dementia. In recounting a story or event patients might back-track, repeating a sentence or whole sequence of sentences verbatim, before continuing. There was no indication that such repetitions were intentional and that the patient was attempting to recap or consolidate information prior to moving on. Indeed, patients showed no apparent awareness of the repetition. Rather, the impression was of "the record being stuck" and of "replaying the same record". Despite back-tracking, patients did not lose train of thought, but could maintain the thread of the story or incident being recounted.

An additional feature, notable in view of patients' severity of illness, was the capacity for self reflection and preservation of awareness of illness, at least to some degree. Patient 1 attempted to rationalise his visual symptoms and to identify factors that gave rise to visual distortions. Patient 2 commented on the oddity of being unable to respond at times. Patient 3 attempted to correct her paraphasic errors. Patient 5 commented on the absurdity of her forgetfulness. It is instructive in this regard that historical reports indicate that initial complaints had in each case been made by the patient, who was aware that things were "not right". At the time of their referral all patients exhibited a similar demeanour, seeming fearful and perplexed. It is tempting to suggest that patients' partial insight into their declining abilities and their vain attempts to comprehend their own predicament might contribute to their state of fearfulness and trepidation.

Recognition of unresponsiveness in CJD is not new. A well recognised feature of SCJD, specified in clinical diagnostic criteria, ${ }^{78}$ is akinetic mutism. This feature, described initially by Cairns and colleagues, ${ }^{22}$ refers to a neurological state in which the patient shows an absence of speech and voluntary movements, and yet appears alert and is able visually to fixate the examiner and may react to auditory stimuli by shifting eye gaze. This alert state of unresponsiveness has been distinguished from the appalic syndrome, ${ }^{23}{ }^{24}$ in which loss of 
responsiveness is associated with disturbance of consciousness. Akinetic mutism is, however, commonly regarded as occurring in late stage disease, and a tacit assumption is that it is either present or absent. The present study suggests that akinetic mutism is not an all-or-none phenomenon. There are gradations in responsiveness. Even at a stage when patients are ostensibly communicative and able to cooperate with cognitive tests, they may show momentary periods of unresponsiveness, sufficient to colour their cognitive performance and to provide a distinctive qualitative performance profile. Emotional salience appeared to have a bearing on patients' responsiveness. During cognitive evaluation a period of unresponsiveness was more easily broken by the introduction of a personally relevant topic than by the presentation of additional tests. It was noted that in late stage disease a patient who was largely unresponsive to hospital staff could engage in a coherent conversation with family members at visiting time. Altered responsiveness appears to be an unusual feature, not seen in other forms of dementia. Patients with frontal cortical disease do show variability in performance, in terms of both response rate and accuracy, ${ }^{25}$ but this is not associated with frank episodes in which the patient appears "switched off" and immobile as in the present cases. It might be speculated that the abnormal language characteristic of back-tracking reminiscent of a "record being stuck", noted in the present series, is also related to patients' variable responsiveness and is a prodrome to a state of akinetic mutism. It might be speculated too that the periodic electroencephalographic changes characteristic of late disease may be associated with disturbances of responsiveness. Video telemetry and prolonged electroencephalographic recording would be required to test this hypothesis.

The variable responsiveness, back-tracking, perseverations, and intrusions in this series of patients suggests that a fundamental impairment is in the activation and regulation of cortical activity from subcortical structures. The pathological analysis of the brains of four patients does not provide direct insight into the underlying mechanisms. Nevertheless, in all patients there was some thalamic involvement and it might be reasonable to speculate that thalamic dysfunction has a contributory role. The thalamus is known to have a regulatory role in the transfer of sensory information to the neocortex and has been implicated in attentional processes, ${ }^{26}{ }^{27}$ particularly in the generation of visual salience. Degeneration of the thalamus has been associated with impairments in vigilance and arousal. ${ }^{28-30}$ Thalamic lesions have been reported to give rise to performance fluctuations, ${ }^{31}$ and "thought disorder", with unpredictible topic shifts unconstrained by contextual factors and intrusions from previous topics. ${ }^{32}$ Thalamic lesions have also been associated with akinetic mutism ${ }^{33}$ and with persistent motor perseveration, ${ }^{34}$ the latter finding being attributed to a disconnection of prefrontal cortico-basal ganglia-thalamo-cortical loops important for the termination of motor plans. Structural brain imaging in SCJD has typically shown high signal changes in the striatum. ${ }^{35-38}$ However, functional imaging using SPECT has shown decreased uptake of tracer in the thalamus. ${ }^{39}$ Taken together these findings are consistent with the notion that in SCJD there is functional disruption to basal ganglia-thalamo-cortical circuitry.

The present series consisted only of four SCJD and two fCJD patients. The duration of illness in three SCJD patients, ranging from nine to 14 months is rather longer than the median of 4.5 months for sCJD. Patient 5 had an atypically young onset. Of the three cases for whom the codon 129 genotype was available two were methionine/valine heterozygous. Thus, the patients are not entirely representative of CJD. There is thus a need for caution in generalising from the present findings to the SCJD or fCJD population as a whole. Nevertheless, the presence of common characteristics in each of a consecutive series of apparently heterogeneous patients suggests that the findings might have some generality (although whether they are more commonly linked to a particular genotypic variant remains open). To our knowledge episodic unresponsiveness and back-tracking phenomena have not been reported in other more common forms of dementia.

The findings of the patients in this study suggest that specific and variable cortical deficits are superimposed on a more pervasive underlying core set of deficits, which are not primarily cortical in origin. Such an interpretation may help to explain the apparent paradox that, despite profound impairment in patients' mental functioning at end stage disease, only limited cortical atrophy is detected at postmortem examination, and brain weight is only minimally reduced. The conventional interpretation is that in a rapidly evolving disease there is simply insufficient time for gross structural changes to develop. An additional contributory factor may be that impairment in cortical function in SCJD and fCJD arises to a large extent secondarily from regulatory failure from subcortical structures.

Episodic unresponsiveness, intrusions, and profound perseveration are characteristic features of the present series of patients. Retrospective analyses of neuropsychological data have inevitable limitations. There is a need for future prospective studies to examine the reproducibility of the present findings and the extent to which they have generality for patients with sCJD. Prospective studies are also required to test the hypothesis that there is a continuum between a state of full, continual responsiveness to a state of akinetic mutism. The question also arises as to the applicability of these features to new variant CJD (vCJD). Reports of vCJD suggest that the clinical presentation differs from that of SCJD, with psychiatric presentations being common. ${ }^{40}{ }^{41}$ Nevertheless, concerted study of neuropsychological characteristics has been limited, a comprehensive neuropsychological evaluation having been published only in a single case.$^{42}$ Moreover, the identification of thalamic changes on magnetic resonance imaging ${ }^{38434}$ points to the importance of thalamic involvement in VCJD, raising the possibility that some overlap in symptomatology might exist.

Authors' affiliations

J S Snowden, D M A Mann, D Neary, Greater Manchester Neuroscience Centre, Hope Hospital, Salford, UK

Competing interests: none declared

\section{REFERENCES}

1 Roos R, Gajdusek DC, Gibbs CJ. The clinical characteristics of transmissible Creutzfeldt-Jakob disease. Brain 1973;96:1-20.

2 Matthews WB. Creutzfeldt-Jakob disease. In: Frederiks JAM, ed. Handbook of clinical neurology: neurobehavioural disorders, Vol. 2 Amsterdam: Elsevier Science Publishers, 1985:46.

3 Will RG, Matthews WB. A retrospective study of Creutzfeldt-Jakob disease in England and Wales 1970-79. i: Clinical features. J Neurol Neurosurg Psychiatry 1984;47:134-40

4 Nevin S, McMenemey WH, Behrman S, et al. Subacute spongiform encephalopathy-a subacute form of encephalopathy attributable to vascular dysfunction (spongiform cerebral atrophy). Brain 1960:83:519-64.

5 Brown P, Cathala F. Creutzfeldt-Jakob disease in France. I. Retrospective study of the Paris area during the ten year period 1968-1977. Ann Neurol 1979:5:189-92.

6 Masters CL, Harris JO, Gajdusek DC, et al. Creutzfeldt-Jakob disease: patterns of worldwide occurrence and the significance of familial and sporadic clustering. Ann Neurol 1979;5:177-88.

7 Cathala F, Brown P, Castaigne P, et al. La maladie de Creutzfeldt-Jakob en France continentale. Etude rétrospective de 1968-1977. Rev Neurol 1979;5:439-54

8 Concerted action of the EU. Surveillance of Creutzfeldt-Jakob disease in the European Community. Minutes of the meeting in Amsterdam, 12-13 June 1998.

9 Mandell AM, Alexander MP, Carpenter S. Creutzfeldt-Jakob disease presenting as an isolated aphasia. Neurology 1989;39:55-8.

10 Kirk A, Ang LC. Unilateral Creutzfeldt-Jakob disease presenting as rapidly progressive aphasia. Can J Neurol Sci 1994;21:350-2.

11 McPherson SE, Kuratani JD, Cummings JL, et al. Creutzfeldt-Jakob disease with mixed transcortical aphasia: insights into echolalia. Behav Neurol 1994;7:197-203. 
12 Vargas ME, Kupersmith M, Savino PJ, et al. Homonymous field defect as the first manifestation of Creutzfeldt-Jakob disease. Am J Ophthalmol 1995; 11 19:497-504

13 Neary D, Snowden JS, Bowen DM, et al. Neuropsychological syndromes in presenile dementia due to cerebral atrophy. J Neurol Neurosurg Psychiatry 1986:49:163-74.

14 Doubleday E, Snowden JS, Varma AR, et al. Qualitative performance characteristics differentiate dementia with Lewy bodies and Alzheimer's disease. J Neurol Neurosurg Psychiatry 2002;72:602-7.

15 Sethi KD, Hess DC. Creutzfeldt-Jakob's disease presenting with ataxia and a movement disorder. Mov Disord 1991;6:157-62.

16 Poon MA, Stuckley S, Storey E. MRI evidence of cerebellar involvement and hippocampal involvement in Creutzfeldt-Jakob disease. Neuroradiology 2001;43:746-9.

17 Tobias E, Mann C, Bone I, et al. A case of Creutzfeldt-Jakob disease presenting with cortical deafness. J Neurol Neurosurg Psychiatry 1994:57:872-3.

18 Cataldi ML, Restivo O, Reggio E, et al. Deafness: an unusual onset of genetic Creutzfeldt-Jakob disease. Neurol Sci 2000;21:53-5.

19 Orimo S, Ozawa E, Uematsu M, et al. A case of Creutzfeldt-Jakob disease presenting with auditory agnosia as an initial manifestation. Eur Neurol 2000;44:256-8

20 Jiang TT, Moses H, Gordon H, et al. Sporadic Creutzfeldt-Jakob disease presenting as major depression. South Med J 1999;92:807-8.

21 Lopez OL, Berthier ML, Backer JT, et al. Creutzfeldt-Jakob disease with features of obsessive-compulsive disorder and anorexia nervosa: the role of cortical-subcortical systems. Neuropsychiatry Neuropsychol Behav Neurol 1997; 10:120-4

22 Cairns H, Oldfield RC, Pennybacker JB, et al. Akinetic mutism with an epidermoid cyst of the 3rd ventricle: with a report on the associated disturbance of brain potentials. Brain 1941;64:273-90.

23 Freemon FR. Akinetic mutism and bilateral anterior cerebral artery occlusion. J Neurol Neurosurg Psychiatry 1971;34:693-8.

24 Otto A, Zerr I, Lantsch M, Weidehaas K, et al. Akinetic mutism as a classification criterion for the diagnosis of Creutzfeldt-Jakob disease. J Neurol Neurosurg Psychiatry 1998;64:524-8.

25 Murtha S, Cismaru R, Waechter R, et al. Increased variability accompanies frontal lobe damage in dementia. JINS 2002;8:360-72.

26 Fimm B, Zahn R, Mull M, et al. Asymmetries of visual attention after circumscribed subcortical vascular lesions. J Neurol Neurosurg Psychiatry $2001 ; 71: 652-7$

27 Michael GA, Boucart M, Degreef JF, et al. The thalamus interrupts top-down attentional control for permitting exploratory shiftings to sensory signals. Neuroreport 2001;12:2041-8.
28 Martin JJ. Thalamic degenerations. In: Vinken PJ, Bruyn GW, eds. Handbook of clinical neurology. Amsterdam: North Holland Publishing Company, 1975;21:587-604

29 Gallassi R, Morreale A, Montagna P, et al. Fatal familial insomnia: behavioral and cognitive features. Neurology 1996;46:935-9.

30 Tihan T, Burger PC, Pomper M et al. Subacute diencephalic angioencephalopathy: biopsy diagnosis and radiological features of a rare entity. Clin Neurol Neurosurg 2001;103:160-7.

31 Mohr JP, Watters WC, Duncan CW. Thalamic hemorrhage and aphasia. Brain Lang 1975;2:3-17.

32 Chatteriee A, Yapundich R, Mennemeier M, et al. Thalamic thought disorder: on being a bit add́led. Cortex 1997;33:419-40.

33 Nagaratnam N, McNeil C, Gilhotra JS. Akinetic mutism and mixed transcortical aphasia following left thalamo-mesencephalic infarction. J Neurol Sci 1999:163:70-3

34 Fung VS, Morris JG, Leicester J, et al. Clonic perseveration following halamofrontal disconnection: a distinctive movement disorder. Mov Disord 1997; 12:378-85.

35 Onofri M, Fulgente T, Gambi D, et al. Early MRI findings in Creutzfeldt-Jakob disease. J Neurol 1993;240:423-6.

36 Barboriak DP, Provenzale JM, Boyko OB. MR diagnosis of Creutzfeldt-Jakob disease: significance of high signal intensity of the basal ganglia. AJR Am J Roentgenol 1994;162:137-40.

37 Yoon SS, Chan S, Chin S, et al. MRI of Creutzfeldt-Jakob disease: asymmetric high signal intensity of the basal ganglia. Neurology 1995:45: 1932-3.

38 Collie DA, Sellar RJ, Zeidler M, et al. MRI of Creutzfeldt-Jakob disease: imaging features and recommended MRI prototcol. Clin Radiol 2001:56:726-39.

39 Matsuda M, Tabata K, Hattori T, et al. Brain SPECT with 1231-IMP for the early diagnosis of Creutzfeldt-Jakob disease. J Neurol Sci $2001 ; 183: 5-12$

40 Zeidler M, Johnstone EC, Bamber RW, et al. New variant Creutzfeldt-Jakob disease: psychiatric features. Lancet 1997:350:908-10.

41 Will RG, Zeidler M, Stewart GE, et al. Diagnosis of new variant Creutzfeldt-Jakob disease. Ann Neurol 2000:47:575-82.

42 Kapur N, Ironside J, Abbott P, et al. A neuropsychologicalneuropathological case study of variant Creutzfeldt-Jakob disease. Neurocase 2001:7:261-7.

43 Coulthard A, Hall K, English PT, et al. Quantitative analysis of MRI signal intensity in new variant Creutzfeldt-Jakob disease. $\mathrm{Br} J$ Radiol signal intensity in

44 Zeidler M, Sellar R, Collie D, et al. The pulvinar sign on magnetic resonance imaging in variant Creutzfeldt-Jakob disease. Lancet 2000;355:1412-18. 